\title{
História do Tempo Presente e América Latina: México - uma entrevista com Eugenia Allier-Montaño
}

\section{Entrevistada}

Eugenia Allier-Montaño es investigadora titular del Instituto de Investigaciones Sociales de la Universidad Nacional Autónoma de México y docente en el Colegio de Estudios Latinoamericanos de la Facultad de Filosofía y Letras de la misma universidad. Doctora en Historia por la Ecole des Hautes Etudes en Sciences Sociales (Francia), realizó una Estancia Posdoctoral en el Instituto de Investigaciones Filosóficas de la unam. Es miembro del Sistema Nacional de Investigadores de México. Su último libro, editado con Emilio Crenzel, es Las luchas por la memoria en América Latina. Historia reciente y violencia política, publicado en inglés como The Struggle for Memory in Latin America. Recent History and Political Violence. Es autora de Batallas por la memoria. Los usos políticos del pasado reciente en Uruguay, que ganó la Mención Honorable del concurso "Mejor libro en Historia Reciente y Memoria 2012" de la Sección "Historia Reciente y Memoria" de la Latin American Studies Association (lasa). Entre otras distinciones, ha sido nombrada en la Cátedra de Estudios del México Contemporáneo de la Universidad de Montreal, Canadá. Actualmente dirige el proyecto de investigación "Hacia una historia del presente mexicano: régimen político y movimientos sociales, 1960-2010".

Entrevista concedida em janeiro de 2019.

\section{Entrevistadoras}

\author{
Elisangela da Silva Machieski \\ Doutoranda no Programa de Pós- \\ Graduação em História da Universidade \\ do Estado de Santa Catarina (UDESC). \\ Florianópolis, SC - BRASIL \\ lismachieski@gmail.com \\ orcid.org/0000-0002-1394-5180
}

\section{Tamy Imai Cenamo}

Mestranda no Programa de Pósgraduação em Historia na Universidad

Nacional Autónoma de México (UNAM).

Cidade do México - MÉXICO tamy.cenamo@gmail.com https://orcid.org/0000-0002-0222-496X

\section{Para citar esta entrevista:}

ALLIER, Eugenia. História do Tempo Presente e América Latina: México - uma entrevista com Eugenia Allier [Entrevista realizada em janeiro, 2019]. Revista Tempo e Argumento, Florianópolis, v. 11, n. 26, p. 601 - 615, jan./abr. 2019. Entrevistadoras: Elisangela da Silva Machieski e Tamy Imai Cenamo. 
Tempo e Argumento: ¿Cuál fue su trayectoria académica desde el inicio de sus estudios universitarios?

Eugenia Allier: De origen tengo dos formaciones paralelas. Al comenzar a estudiar la licenciatura opté por una doble formación. En primer lugar, la carrera de Psicología Social en la Universidad Autónoma Metropolitana de la Ciudad de México. Y en segundo término, la licenciatura en Estudios Latinoamericanos en la Universidad Nacional Autónoma de México (UNAM). Esas dos formaciones determinaron bastante el tipo de temáticas que me ha interesado estudiar: la historia desde una perspectiva reciente y las cuestiones sociales vinculadas con la violencia y lo traumático. Después, a nivel posgrado continué con ambos intereses. Realicé una maestría en psiconálisis en México y una maestría en Historia en Francia. Fue en Francia donde llevé a cabo mis estudios de doctorado en la Escuela de Altos Estudios en Ciencias Sociales, iguElial que la maestría, en Historia.

En toda esa formación académica que tuve en México y Francia mis preocupaciones se fueron decantando por la historia del presente, la violencia política, lo traumático y la memoria colectiva. En mis primeros años estudié el caso uruguayo, desde la transición democrática en la licenciatura hasta las batallas por la memoria del pasado reciente (dictadura civico-militar) en el espacio público. En todo ello siempre ha habido un interés teórico muy importante: debatir conceptos y pensar categorías. Por eso amplié los casos estudiados y al comenzar una estancia posdoctoral en el Instituto de Investigaciones Filosóficas de la UNAM decidí empezar a trabajar sobre México, en particular acerca del movimiento estudiantil de 1968, un tema que siempre me ha apasionado y que de alguna manera ha acompañado mi vivencia personal (digamos que pertenezco a la generación "hija del 68").

Creo que fue gracias en parte a esa formación interdisciplinaria que pude encontrar temas de estudio que hacen converger mis diferentes intereses. $Y$ es que una de las características más importantes de la historia del tiempo presente es justamente su carácter interdisciplinario: hay historiadores, sociólogos, antropólogos, politólogos y psicólogos trabajando en este ya no tan nuevo campo de estudio. Seguramente 
también fue por esa formación que pude entrar a trabajar al Instituto de Investigaciones Sociales de la UNAM (IIS-UNAM), que se caracteriza por contar entre sus filas a sociólogos, politólogos, historiadores, antropólogos. En este Instituto lo fundamental es el presente en el que vivimos, aunque cada disciplina define de manera diferente este tiempo histórico. En ese sentido, debo decir que soy muy feliz de trabajar en el IIS-UNAM: puedo dedicar la mayor parte de mis esfuerzos a la investigación y la docencia, tengo colegas de distintas disciplinas con quienes discutir, y puedo consagrarme a pensar teóricamente así como a aplicar las categorías analíicas que me interesan a distintos casos empíricos. Sigo interesada en América Latina, trabajo mucho sobre México; avanzo en el estudio de los movimientos estudiantiles, pero a la par voy trabajando en movimientos armados, violencia política, violencia de Estado, memorias públicas, lugares de memoria, y todo ellos enmarcado en la historia del tiempo presente.

Tempo e Argumento: ¿Cómo podría situar su producción académica dentro del escenario historiográfico de México?

Eugenia Allier: En México, una parte de la academia histórica mantiene una forma un poco tradicional de hacer historia: ${ }^{1}$ se sigue estudiando el pasado más lejano, las temáticas se han movido poco respecto a lo que se hacía en el siglo pasado, y se rechaza el uso de nuevas fuentes. Por supuesto, no existe una única forma de hacer historia en México: la disciplina histórica no es un conjunto homogéneo, pero sí hay líneas dominantes.

Evidentemente, en los últimos años ha habido transformaciones importantes y han surgido nuevas temáticas, como un fuerte campo en la historia de género (de las mujeres), en la vida cotidiana, en la infancia. Además, la historia oral se ha desarrollado fuertemente en muchas instituciones, lo mismo que la historia visual

\footnotetext{
1 Pienso aquí en el libro de Peter Burke, Formas de hacer historia (Madrid, Alianza Editorial, 2003) y en las discusiones sobre la nueva historia y la historia tradicional: si bien no se trata realmente de campos bien definidos, la "nueva historia" haría referencia a aquellas formas que surgieron en los años 1970-1980 y que transformaron las temáticas y los periodos estudiados, así como las fuentes para su estudio.
} 
vinculada con la fotografía. También están los trabajos sobre descolonización. Hay, pues, renovación en la producción historiográfica en México. Pero se trata de una renovación que, como casi siempre, viene de los márgenes y tiene dificultades para alcanzar el centro.

Considero que mi trabajo historiográfico se enmarca en tres grandes rubros: la historia del presente, la historia de la memoria y la historia oral. En la historia oral México ya comienza a tener una tradición importante: no quiero olvidar a nadie, pero está el trabajo de Graciela de Garay, Patricia Pensado y Silvia Dutrénit desde el Instituto Mora, de Gerardo Necoechea y Mario Camarena en la Escuela Nacional de Antropología e Historia, y tantos otros en distintos estados del país. Respecto a la memoria, también hay trabajos importantes que se vinculan con memorias nacionales, conmemoraciones; pienso en el trabajo de Virginia Guedea, y en un ámbito muy diferente en el de Mario Rufer, sólo por citar algunos ejemplos. Quizás el ámbito en el que apenas comienza a generarse una producción importante es en el de la historia del presente que, como veremos, es un campo en expansión. Hay antecedentes importantes también en ese sentido, como Pablo Yankelevich y el trabajo sobre el exilio argentino en México, ${ }^{2}$ la propia Silvia Dutrénit ${ }^{3}$ y los estudios sobre transiciones y exilio, y la labor de Graciela de Garay. Pero ellos no conformaron un campo específico de la historia del presente en el país. Se trató más de trabajos excepcionales pero individuales.

\section{Tempo e Argumento: Actualmente, ¿cuáles son los enfoques de sus trabajos?}

Eugenia Allier: El año pasado concluí un libro sobre las memorias públicas del movimiento estudiantil de 1968 en México: una historia que abarca cincuenta años, de 1968 a 2018 y revisa los cambios y permanencias en las formas de recordar el 68 en el país. Con ese libro estoy cerrando un ciclo sobre mi trabajo con memorias públicas. Se trata de una labor que llevo más de veinte años desarrollando y que fue desde la dictadura uruguaya hasta el 68 mexicano.

\footnotetext{
2 Pablo Yankelevich, Ráfagas de un exilio: argentinos en México, 1974-1983. México: COLMEX, 2009.

3 Silvia Dutrénit, Aquellos niños del exilio. Cotidianidades entre el Cono Sur y México, México, 2015.
} 
En el aspecto empírico, ahora he comenzado tres nuevas líneas de investigación. En primer lugar, una biografía política. Vincula mis intereses previos, con el interés por la escritura de una historia individual en su vínculo con la historia social. Y en segundo término, y de alguna manera ligado al interior, historias de vida de militantes en los años 1970: se trata de ver lo individual, pero a través de una generación. Por último, un interés personal acerca de los lugares de memoria de violencia política y sus relaciones con el turismo.

A nivel teórico continúo con el interés por estos lugares de memoria, pero ahora desde su vinculación con el turismo, eso que se ha llamado "turismo de trauma". ¿Por qué los lugares donde se vivieron tragedias se han convertido en espacios visitados por los turistas? ¿Qué tipo de turistas los visitan? El otro gran tema, que no he dejado de pensar en los últimos años es justamente el de la historia del tiempo presente. En este momento estoy realizando la publicación de un libro editado sobre historia del presente, en el que participarán importantes especialistas de América Latina y Europa: definiciones, debates, metodologías, fuentes, temáticas.

Tempo e Argumento: ¿Cómo fue que la Historia del Tiempo Presente se volvió una preocupación en su carrera? ¿Cuáles fueron las referencias teórico-metodológicas que la influyeron en su proyecto de historizar el "tiempo presente"?

Eugenia Allier: Yo puedo percibir dos aspectos que definieron mi interés por la historia del tiempo presente. En primer lugar, las circunstancias sociales y políticas en las que crecía. Mi infancia transcurrió en los años 1970 en la Ciudad de México. Tal y como lo muestra una amplia historiografía contemporánea, dentro de América Latina México se convirtió en uno de los destinos elegidos por un amplio sector del exilio latinoamericano de esos años. A la par que el gobierno mexicano reprimía fuertemente a diversos grupos de opositores nacionales, abría las puertas a los

\footnotetext{
${ }^{4}$ Un ejemplo de ello es mi reciente trabajo: Eugenia Allier-Montaño, "Tlatelolco, lugar de memoria y sitio de turismo. Miradas desde el 68”, en Revista Mexicana de Ciencia Política nueva época, año LXIII, núm. 234, septiembre-diciembre de 2018, pp. 215-238.
} 
perseguidos de Latinoamérica. Por azares del destino, durante mi formación intermedia (secundaria y preparatoria, entre los 12 y los 18 años), acudí a una escuela Ilamada Centro Escolar Hermanos Revueltas. Ahí estudiaron también muchos hijos de exiliados políticos: uruguayos, argentinos, haitianos, salvadoreños, chilenos. De manera que el peso del pasado reciente en mi formación política y sentimental fue muy grande. El presente estaba a mi alcance.

En segundo lugar, la propia formación que tuve en las licenciaturas me reafirmó ese interés por el presente latinoamericano: seguramente ello influyó en mi decisión de cursar la carrera de Estudios Latinoamericanos. Mis temas de interés historiográfico siempre se vincularon con el presente. Y cuando llegue a Francia, pude darle nombre y lugar en un campo a mis intereses: la historia del tiempo presente.

En la Escuela de Altos Estudios en Ciencias Sociales no existe un espacio o cátedra especializada en la historia del presente, pero están algunos de los autores que, de alguna manera, influyeron en su conformación. Tuve la oportunidad de estudiar con Pierre Nora y de escuchar sobre los lugares de memoria que no son una forma de historizar el presente, ${ }^{5}$ con Philippe Joutard y su aproximación a la historia oral. ${ }^{6} \mathrm{Y}$ sobre todo, pude acercarme al Institute d'Histoire du Temps Présent (IHTP), creado en los años 1970, pilar en la discusión y escritura de la historia del presente. El primer libro que leí sobre este campo historiográfico era justamente una edición de 1993 del IHTP que reunía varias decenas de ponencias de un coloquio que se había desarrollado en torno al tema: Écrire l'histoire du temps présent.7 Me sirvió muchísimo, no sólo para comprender que el campo existía y era reconocido, sino para conocer los debates en torno a las críticas sobre la historia del presente, sus límites y sus fortalezas. No encontré ahí, sin embargo, una definición sobre lo que era el tiempo presente. Pero en esa época pude acercarme a relevantes historiadores del campo, conocer sus obras y acercamientos al presente.

\footnotetext{
5 Pierre Nora, “Entre memoria e historia. La problemática de los lugares”. En Pierre Nora, en Les Lieux de mémoire, Montevideo: Ediciones Trilce, 2008.

${ }^{6}$ Philippe Joutard, Esas voces que nos llegan del pasado. México: Fondo de Cultura Económica, 1999. Philippe Joutard, Histoire et mémoires, conflits et alliance. Paris: La Découverte, 2013.

${ }^{7}$ Ecrire l'histoire du temps présent. 1993. París: CNRS.
} 
Probablemente quien más me influyó en ese sentido fue Henry Rousso, del IHTP: su texto sobre la historización de las memorias de Vichy me impactó fuertemente, ${ }^{8}$ y lo utilicé de modelo para hacer mi tesis de doctorado sobre Uruguay y las memorias de la dictadura cívico-militar. 9 Después he tenido el privilegio de poder seguir discutiendo con él en diversos espacios, así como con Frédérique Langue, que tiene muy importantes trabajos empíricos sobre el presente latinoamericano. ${ }^{10}$

Debieron pasar varios años y una renovación de mi interés por pensar en la definición del tiempo presente, para encontrar otra referencia fundamental: el libro de Julio Aróstegui, La historia vivida. ${ }^{11}$ Creo que se trata de uno de los aportes más lúcidos y fundamentales para pensar esta parcela historiográfica. En este texto, Aróstegui no sólo hace una genealogía del campo historiográfico del presente a través de sus distintos países e instituciones, sino que muestra las diferentes definiciones y formas de trabajo existente. Por si ello fuera poco, presenta su propia definición: el de una historia de la coetaneidad del historiador; para Aróstegui, historizar el presente es hacer la historia vivida por la generación del historiador que escribe. En ello yo tengo mis diferencias: la historia no se puede definir por el historiador, sino por las temáticas y abordajes. A partir de su propuesta, yo considero que podemos pensar la historia del presente como aquella que estudia al menos una de las tres generaciones que conocieron un acontecimiento.

A la par fui leyendo a algunos autores latinoamericanos, como María Inés Mudrovcic, quien desde la filosofía ha hecho aportes muy importantes, ${ }^{12}$ Hugo Fazio $^{13}$ y por supuesto, los aportes desde el campo de la historia reciente, que no es exactamente igual al de la historia del presente. En ese sentido, el libro de Marina Franco, con quien estudié y debatí en Francia, y Florencia Levín, es muy importante

\footnotetext{
${ }^{8}$ Éric Conan, Henry Rousso, Vichy, un passé qui ne passe pas, París, Gallimard, 1996.

9 Eugenia Allier Montaño (2010). Batallas por la memoria. Los usos políticos del pasado reciente en Uruguay. Montevideo: Trilce / Instituto de Investigaciones Sociales-UNAM.

${ }^{10}$ Luc Capdevilla y Frédérique Langue (eds.), Le passé des émotions d'une histoire à vif, Amérique Latine et Espagne, Rennes, Presses Universitaires de Rennes, 2009.

11 Julio Aróstegui, 2004. La historia vivida. Sobre la historia del presente. Madrid: Alianza Editorial.

${ }^{12}$ María Inés Mudrovcic, Historia, narración y memoria. Escritos sobre filosofía de la historia, AKAL, Madrid, 2005.

${ }^{13}$ Hugo Fazio, 2010. La historia del tiempo presente: historiografía, problemas y métodos. Bogotá: Universidad de los Andes.
} 
para la conformación del campo en el Cono Sur. ${ }^{14}$ Por supuesto, el debate en América Latina sigue creciendo y hay cada vez más discusiones y contribuciones para pensar teóricamente esta parcela historiográfica.

Tempo e Argumento: En el Brasil, durante algún tiempo, el campo de la historia del tiempo presente fue blanco de críticas, sobre todo en cuanto a los procedimientos del hacer historiográfico que implica. ¿Cómo se constituyó ese campo del saber en México? ¿Cuáles son los principales temas de investigación por los historiadores del "tiempo presente" en su país?

Eugenia Allier: Como decía en otro momento, el campo de la disciplina histórica en México ha sido un poco reacio a incluir nuevas formas de hacer historia. Ciertas temáticas, como el género, la infancia, las mentalidades, la memoria, han sido paulatinamente aceptadas. Las aproximaciones metodológicas han tenido más dificultades para hacerse un lugar: es el caso especialmente de la historia oral.

La historia del presente desafía diversos aspectos de la historia tradicional: no sólo las temáticas, no sólo las formas metodológicas de investigar y escribir la historia, sino (lo que parece más grave a ojos de algunos historiadores) que cuestiona el tiempo de la historia. No se trata únicamente por ejemplo de hacer entrevistas a mujeres e incluirlas en el tiempo, se trata de integrar el presente (desterrado de la historia durante el siglo XIX) a los tiempos históricos. Es algo que sobrepasa la tolerancia de muchos historiadores.

Por todo ello, la constitución de un campo historiográfico específico de la historia del tiempo presente en México ha sido un gran reto. En la generación anterior a la mía hubo importantes historiadores que se interesaron por el presente vivido: ya hablamos de Pablo Yankelevich, Silvia Dutrénit, Graciela de Garay. Pero como mencioné, no se constituyó un campo: con vínculos, debates, intercambios. En mi generación también ha habido historiadores muy importante que han estudiado periodos más cercanos, como Ariel Rodríguez Kuri, Verónica Oikión, Alberto del

\footnotetext{
${ }^{14}$ Marina Franco y Florencia Levín (comps.). 2007. Historia reciente. Perspectivas y desafíos para un campo en construcción. Buenos Aires: Paidós,
} 
Castillo. $Y$ es un poco con ellos, y sobre todo con muchos alumnos que ellos han formado, que se está creando el campo.

Nosotros iniciamos en el Instituto de Investigaciones Sociales de la UNAM un seminario hace unos ocho años. Muchos jóvenes historiadores de una nueva generación han pasado por ahí para discutir sus trabajos y generar esos debates, intercambios y vínculos que supone un campo: Camilo Vicente, César Vilchis, Rodolfo Gamiño, Aleida García Aguirre, Sergio Arturo Sánchez Parra, Sara Musotti, Rogelio Ruiz, Alicia de los Ríos; también hemos recibido colegas latinoamericanos pertenecientes al campo y que nos ayudan a abrir nuestras perspectivas: Soledad Lastra, Emilio Crenzel, María Inés Mudrovcic. Es el empuje de esta nueva generación, aunado a los aportes de las anteriores, el que está forjando el campo de la historia del presente en México.

En los últimos años se han creado diversos seminarios sobre historia del tiempo presente, a la par que se desarrollan proyectos de investigación sobre la temática en la Universidad Iberoamericana, el Instituto Mora, en la Facultad de Filosofía y Letras de la UNAM, en la Escuela Nacional de Antropología e Historia. Ahora el Instituto Nacional de Estudios Históricos de las Revoluciones en México también está por abrir un seminario de historia del presente. A la par de todo este trabajo académico, creo que el interés del actual gobierno de Andrés Manuel López Obrador por la violencia, los desaparecidos y los muertos, va a poner en el centro del debate público nuestra historia reciente, las memorias, la oralidad y, por supuesto, el presente.

Tempo e Argumento: En términos historiográficos, ¿existen diferencias entre la Historia del Tiempo Presente producida en México y en otros países latinoamericanos?

Eugenia Allier: Definitivamente creo que hay especificidades importantes del campo en cada caso nacional. Diversos especialistas han mostrado cómo cada parcela historiográfica se ha conformado según las circunstancias históricas y los contextos sociales, económicos y políticos nacionales. Si en muchos países, la "última 
catástrofe" o "tragedia", para retomar el término de Henry Rousso, ${ }^{15}$ define el presente, las circunstancias de México han conllevado una experiencia especial.

Ya hablamos de las dificultades para crear el campo debido a las formas conocidas por la disciplina histórica en México. A ello hay que sumarle las particularidades sociales y políticas. México no conoció una dictadura militar formal ni una guerra civil como las que asolaron la región latinoamericana en los años 1970 y 1980 . No hubo un periodo de terrorismo de Estado. Pero ello no implica que no hubiese graves violaciones de derechos humanos. Si bien está pendiente la historización de las violencias política y de Estado en México, ya podemos señalar que desde fines de los años 1950 y hasta el día de hoy no ha dejado de ejercerse la violencia de Estado, simplemente que en cada periodo se ha ejercido bajo distintas modalidades.

Todo lo anterior ha implicado dos variantes importantes en la conformación de la historia del presente en México. En primer lugar, que a diferencia de otros países (como Francia y Argentina), la historia del tiempo presente en México no es un periodo histórico: no se trata del estudio del "último trauma", ni de la última dictadura militar. En México, el presente es una categoría, no un tiempo.

Decía anteriormente que en mi propia definición de tiempo presente recupero el trabajo de Julio Aróstegui, pero adecuándolo a las temáticas y no al historiador. Cuando el historiador estudia un periodo del cual existe al menos una de las tres generaciones ${ }^{16}$ que vivieron un acontecimiento se está haciendo una historia de la coetaneidad, de un tiempo que aún es vigente, porque el historiador está investigando un presente histórico: un presente del cual es coetáneo, al ser coetáneo de al menos una de las generaciones que lo vivieron. El presente histórico entonces no es el ahora o la inmediatez, sino un lapso de tiempo más amplio que

\footnotetext{
${ }^{15}$ Ver, por ejemplo, su libro más reciente sobre el tema: Henry Rousso, La dernière catastrophe. L'histoire, le présent, le contemporain. Paris, Gallimard, 2013.

${ }^{16}$ En cada momento histórico existen tres generaciones que comparten un momento histórico: la generación en formación (sucesora), aquella que iría más o menos de los 0 a los 30 años, y que justamente se caracteriza por estar formándose; la generación hegemónica (activa), entre 30 y 60 años, y que detenta tanto los medios de producción como el poder político, administrativo y social; la generación transmisora (antecesora), más allá de los 60-70 años, aquella que ya no detenta los medios, pero que aún tiene poder a su alcance y que en muchos sentidos está transmitiendo sus conocimientos y su poder a las otras dos generaciones.
} 
está vinculado con la existencia de las generaciones que experimentaron un suceso. La experiencia de aquellos que vivieron un acontecimiento prefigura el presente en el que los coetáneos siguen viviendo. Por eso es que decimos que la historia del tiempo presente tiene márgenes móviles. No es un periodo ni un acontecimiento, es una historia que se liga con la coetaneidad y con las generaciones vivas que experimentan el tiempo histórico. Por eso se va moviendo con los propios límites de lo contemporáneo-coetáneo.

En segundo término, las condiciones sociales y políticas en México han implicado que las temáticas estén menos dirigidas por la violencia política que en otros lugares. Si bien el movimiento estudiantil de 1968 y la represión sufrida desde el Estado ha sido centro de una historiografía del presente, así como más recientemente lo referido a la llamada Guerra Sucia del Estado en contra de los movimientos sociales en los años 1970-1990 y la historia de los movimientos armados, en México también se ven aportes importantes desde otras temáticas. La memoria en el país estudia también las conmemoraciones del Bicentenario de la Independencia y el Centenario de la Revolución, la conformación de espacios virtuales en Internet, las agrupaciones y definiciones de grupos de homosexuales y lesbianas, la ecología. En fin, temas y temporalidades que normalmente no están presentes en los campos de la historia del presente en otros países (a excepción de Brasil).

En síntesis, se trata de un campo formado con relativo atraso respecto a Francia y el Cono Sur, pero que está más abierto a nuevos enfoques, temáticas y temporalidades.

Tempo e Argumento: En 2018 se conmemoraron los 50 años del 68. Usted organizó un evento internacional sobre el tema, ¿Cuál es la importancia de este acontecimiento en la reflexión sobre el pasado reciente de México?

Eugenia Allier: Muchos historiadores, como Henry Rousso, Frédérique Langue, Marina Franco, María Inés Mudrovcic, han planteado que la historia del presente (o historia 
reciente) se conforma en cada país por el peso de la última tragedia vivida. Como ya señalé, no estoy segura de que sea así en todos los casos. En México no se vivió una dictadura formal, tampoco hubo una época de terrorismo de Estado. Y probablemente, como dije, eso nos ha llevado a conformar un campo de la historia del presente distinto al de otros países.

Aclarado eso, tal vez el epicentro del pasado reciente en México sea el movimiento estudiantil de 1968. Uno de los propósitos de mi trabajo ha sido mostrar cómo tanto desde la memoria como desde la historia, el 68 se ha convertido en el acontecimiento más relevante en la segunda mitad del siglo XX.

A partir del año 2000, se puede observar un consenso político-social en el país sobre el lugar que ocupa el movimiento estudiantil de 1968 en la historia nacional reciente: se le considera un "parteaguas" vinculado con la lucha por la democracia. Además, en los últimos diez años se ha conocido una institucionalización del movimiento estudiantil: ha pasado a formar parte de la historia oficial del país y es reconocido como un referente histórico para la segunda mitad del siglo XX. En 2002, integró los acontecimientos que los niños de primaria revisan a través de los Libros de Texto Gratuitos; en 2007, se inauguró el museo Memorial del 68 en la UNAM; en 2008, se modificó el artículo 18 de la Ley sobre el Escudo, la Bandera y el Himno Nacionales, estableciendo como fecha solemne nacional el día 2 de octubre: “Aniversario de las víctimas en la lucha por la democracia de la Plaza de las Tres Culturas en Tlatelolco, en 1968": desde entonces, la bandera nacional debe ondear a media asta en los recintos institucionales del país cada 2 de octubre. ${ }^{17}$

A la par que iba obteniendo este consenso, el 68 fue sufriendo una mitificación por parte de muchos actores políticos y sociales. No obstante, pese al consenso y a la mitificación de que ha sido objeto el movimiento estudiantil, los grupos de víctimas encabezados por el Comité 68 Pro Libertades Democráticas no han conseguido dos de sus principales objetivos: el esclarecimiento de lo ocurrido el 2 de octubre y el

\footnotetext{
17 El 2 de octubre de 1968, las fuerzas policiacas del entonces presidente mexicano Gustavo Díaz Ordaz reprimieron una manifestación política estudiantil que se llevaba a cabo en la Plaza de las Tres Culturas en Tlatelolco, Ciudad de México. Decenas de estudiantes fueron asesinados y tantos más quedaron heridos o ilegalmente detenidos, razón por la cual dicho acontecimiento recibió el nombre de La Masacre de Tlatelolco.
} 
enjuiciamiento y castigo a los responsables por la violencia de Estado. A diferencia de lo que sucede en muchos países latinoamericanos, en México no hay lucha por la apropiación de los sentidos memoriales respecto al movimiento del 68: la mayor parte de los actores políticos y sociales acuerdan que el 68 fue un movimiento que luchó por libertades democráticas y fue violentamente reprimido. Y si varios países de la región han obtenido la creación de comisiones de verdad para desentrañar lo acontecido y han logrado llevar a juicio y a la cárcel a muchos responsables de crímenes de lesa humanidad, en México los intentos por aclarar la violencia de Estado no han tenido logros sustanciales y el juicio por el 2 de octubre fue resuelto a favor de los principales acusados. ¿Significa que el consenso conlleva impunidad? Probablemente no en todos los casos, pero el régimen del Partido Revolucionario Institucional (PRI) utilizó durante muchos años el mecanismo de la "cooptación": incorporar los discursos y actores al sistema para, de cierta manera, neutralizarlos políticamente. Ese parece que ha sido el resultado de las luchas por obtener reconocimiento respecto al movimiento estudiantil de 1968.

En cualquier caso, 2018 fue la consagración definitiva del movimiento estudiantil en tanto eje central del pasado reciente de México: el acontecimiento que marca un parteaguas en la historia política y social después de la Revolución Mexicana (19101917). En 2018 se conmemoraron 50 años del movimiento estudiantil de 1968 en México. Esta conmemoración coincidió, por primera vez, con un cambio político. Un momento rupturista en el que la izquierda mexicana llegó al poder, lista para asistir a la conmemoración anual del 2 de octubre, la masacre de Tlatelolco, la que año con año reúne a buena parte de la sociedad al grito de "2 de octubre no se olvida". A cincuenta años, los actores políticos, sociales y culturales se movilizaron para festejar el medio siglo del movimiento: ese año, a lo largo y ancho del país tuvieron lugar al menos unas cinco decenas de coloquios, seminarios y talleres, se publicaron más de ochenta libros, revistas especializadas y revistas político-culturales, se inauguraron más de veinte muestras artísticas, se dieron a conocer cerca de diez documentales y series de televisión, se llevaron a cabo diversas manifestaciones artísticas, y se realizaron marchas conmemorativas en la Ciudad de México y en 
otros 13 estados del país; todo tipo de producción cultural y académica fue utilizada para la ocasión.

Los sentidos del pasado y del presente fueron activados en las celebraciones. Por un lado, se le consagró el lugar central en la historia política y social nacional. Las ceremonias en la Universidad Nacional Autónoma de México (UNAM), en el Instituto Politécnico Nacional (IPN) y en las Cámaras de Diputados y Senadores, así como la resolución de la Comisión Ejecutiva de Atención a Víctimas (CEAV) de reconocer la Masacre de Tlatelco como crimen de Estado muestran que el 2 de octubre ha sido ya incorporado a la historia nacional como un día muy grave en la violación de los derechos humanos de los mexicanos, mientras el movimiento en su conjunto es entendido como un hito en la lucha por la democracia del país.

Pero las conmemoraciones también son un termómetro para conocer el presente. Y en ese sentido, hay ejemplos de cómo la actualidad echa nueva luz sobre el pasado. Por un lado a través de la reinaguración del museo Memorial del 68 que si bien tiene como centro el 68, pone un énfasis especial en los movimientos sociales y armados que le siguieron, en una línea histórica que nos lleva al presente de democracia y elecciones que se vivía en 2018, con la histórica elección presidencial del candidato del Movimiento de Renovación Nacional (MORENA), Andrés Manuel López Obrador. Por el otro lado, el nuevo movimiento feminista en México ha hecho que la voz de las mujeres se vuelva más fuerte y audible: en múltiples foros académicos y sociales se recalcó que, durante muchos años, los varones participantes en el movimiento habían tenido la voz cantante en las narraciones sobre el pasado, que habían sido ellos quienes habían dado las pautas para interpretar lo ocurrido en 1968. Frente a ello, hubo múltiples eventos en los que se dio la voz a las mujeres, eventos llenos a rabiar, en los que el centro de la atención fueron las mujeres. Y eso lo pudimos ver con el libro publicado por Ana Ignacia Rodríguez Márquez “La Nacha" en donde narra una parte de su vida dura y significativa: la cárcel política que conoció tras el movimiento estudiantil. ${ }^{18}$

\footnotetext{
${ }^{18}$ Ana Ignacia Rodríguez Márquez, Cartas de libertad. México: Quinto Sol, 2018.
} 
2018 significó, entonces, la instituciónalización definitiva del movimiento estudiantil en la historia política y social de México. Significó también la apertura a nuevas lecturas y resignificaciones, ligadas con el presente político democrático del país. Y significó también la apertura a nuevas voces, particularmente las de las mujeres, que de esa manera, dieron a conocer sus puntos de vista y testimonios sobre aquel mítico año.

En síntesis, la historia de 1968 nos permite conocer y reconocer nuestro pasado como nación: las movilizaciones sociales de los años 1960 (anteriores y posteriores), la posición de la población frente a lo político, y la violencia de Estado en contra de los opositores políticos. Pero al mismo tiempo, la historia de las memorias públicas del movimiento estudiantil nos permite conocer nuestros distintos presentes políticos: cada nueva lectura del 68 mucho nos dice de la coyuntura política, económica y social desde la que se está hablando. Narrar el pasado es mirar el presente. El 68 ocupa un lugar central en el pasado reciente de México, y nos permite explicar y comprender el presente en el que vivimos, con todas sus complejidades y violencias. En ese sentido, tenemos que continuar estudiando la historia de las violencias de Estado en nuestro país, y el lugar que en ella ocupa el 2 de octubre de 1968 con la masacre en la Plaza de las Tres Culturas de Tlatelolco.

Universidade do Estado de Santa Catarina - UDESC

Programa de Pós-Graduação em História - PPGH

Revista Tempo e Argumento

Volume 11- Número 26 - Ano 2019 tempoeargumento@gmail.com 